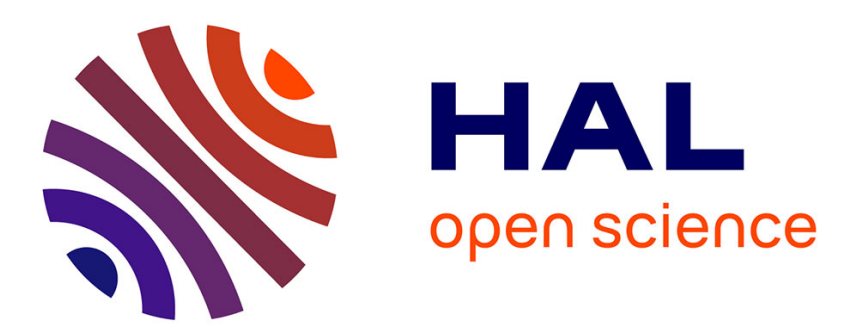

\title{
Experimental determination and simulation of the angular distribution of the metal flux during magnetron sputter deposition
}

M Horkel, K van Aeken, C Eisenmenger-Sittner, D Depla, S Mahieu, W P Leroy

\section{To cite this version:}

M Horkel, K van Aeken, C Eisenmenger-Sittner, D Depla, S Mahieu, et al.. Experimental determination and simulation of the angular distribution of the metal flux during magnetron sputter deposition. Journal of Physics D: Applied Physics, 2010, 43 (7), pp.75302. 10.1088/0022-3727/43/7/075302 . hal-00569764

\section{HAL Id: hal-00569764 \\ https://hal.science/hal-00569764}

Submitted on 25 Feb 2011

HAL is a multi-disciplinary open access archive for the deposit and dissemination of scientific research documents, whether they are published or not. The documents may come from teaching and research institutions in France or abroad, or from public or private research centers.
L'archive ouverte pluridisciplinaire HAL, est destinée au dépôt et à la diffusion de documents scientifiques de niveau recherche, publiés ou non, émanant des établissements d'enseignement et de recherche français ou étrangers, des laboratoires publics ou privés. 


\title{
Experimental determination and simulation of the angular distribution of the metal flux during magnetron sputter deposition
}

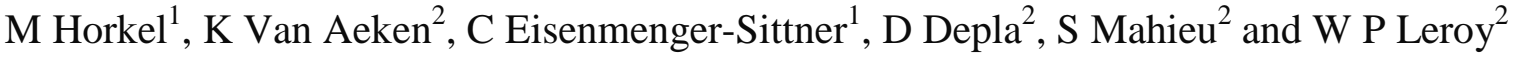 \\ ${ }^{1}$ Institute of Solid State Physics, E-138, Vienna University of Technology, Wiedner \\ Hauptstrasse 8-10, Vienna A-1040, Austria \\ ${ }^{2}$ Department for Solid State Sciences, Ghent University, Krijgslaan 281 (S1) 9000, Ghent \\ 9000, Belgium
}

e-mail:m.horkel@gmx.at

To understand the film growth during magnetron sputter deposition a detailed knowledge of the flux of sputtered species from the target towards the substrate is vital. One important parameter is the angular distribution of the impinging neutral target atoms on the substrate, since it is responsible for e.g. self shadowing effects. The determination of the angular distribution of the metal flux at an arbitrary point in the deposition chamber is achieved by a pinhole-camera, where the information of the angular distribution is converted into a thickness profile. This paper describes the construction of such a pinhole-camera which is capable of differential pumping, the determination of the angular distribution for a wide variety of target materials, and which can easily be inserted into a deposition chamber. The angular distributions of different materials $(\mathrm{Cu} ; \mathrm{W} ; \mathrm{Al} ; \mathrm{Ti} ; \mathrm{Mg})$ at different parameters (pressure, lateral position, and vertical position) are experimentally determined and compared to simulations obtained from a newly developed Monte Carlo code. It was also investigated, if 
parameters derived from the angular distribution are related to the degree of thermalization of the impinging particles.

PACS: 81.15.Cd Deposition by sputtering

Keywords: angular distribution, sputter deposition, magnetron deposition, pinhole camera, thermalization

Submitted to: Journal of Physics D: Applied Physics

\section{Introduction:}

The angular distribution of the impinging neutral particles during magnetron sputtering, one of today's most important deposition techniques, is an interesting parameter. First the angular distribution may have a direct relationship with the properties of the deposited layers, e.g. the porosity of the film caused by self shadowing effects [1, 2], mound formation [3], the step coverage in microelectronics [4], or the complex film structure caused by glancing angle deposition [5]. Beside its known influences, the angular distribution is an important parameter for the simulation of film growth $[6,7]$. In recently developed techniques like the deposition of biaxially aligned layers [8,9], the angle of the impinging atoms is even a vital parameter. More over, a detailed study of the angular distribution can provide insight in the fundamental principles of magnetron sputter deposition, such as the initial angular distribution of the sputtered particles, the shape of the local sputter rate and transport of the sputtered particles through the gas phase as these all influence the angular distribution. In this context, a key aspect of magnetron sputter deposition is the energy of the particles arriving at the substrate which is substantially higher than thermal energy. Of course this latter has its impact on the thin film growth $[8,9]$, and hence the degree of thermalization of the arriving flux is an important issue. As the angular distribution is influenced, together with the energy of the particles, by the gas transport, measurements of the angular distribution can give insight in the thermalization process. 


\section{Determination and simulation of the angular distribution of the metal flux}

To measure the angular distribution of the impinging particles, a device called Metal Flux Monitor (MFM) has been constructed. It is a pinhole camera [10-15], which converts the angular distribution of the arriving flux into a thickness profile of a layer on a substrate behind a pinhole. This information can briefly be described as an image of the local sputter rate multiplied by the initial angular distribution, which is then blurred by gas phase scattering. One difference of the MFM to the pinhole cameras in the previous papers $[12,13]$ is that it can be inserted via a load lock and is not fixedly mounted in the deposition chamber. This allows a swift change of the position of the MFM, and a high experimental throughput. The methods to analyze the thickness profiles were also designed in respect to the high experimental throughput and allow a large variety of materials (not only metallic but also transparent materials) to be measured. The experiments were performed for various materials $(\mathrm{Cu} ; \mathrm{W}$; $\mathrm{Al} ; \mathrm{Mg} ; \mathrm{Ti}$ ) at different parameters (lateral position; distance magnetron-MFM; pressure), and the results are compared to simulations by the Monte Carlo (MC) code SIMTRA [16].

Section 2 of this paper describes the design of the MFM, its fundamental principles and the determination of the thickness profiles via optical methods. The experimental setup and parameters are given in Section 3, while Section 4 gives a brief description of the MC code SIMTRA. The experimental results in comparison to the simulations performed by SIMTRA are presented in Section 5. A relationship between the degree of thermalization and the properties of the obtained angular distribution was studied.

\section{Metal Flux Monitor}

The Metal Flux Monitor (MFM) is a pinhole camera which converts the information of the angular distribution of the metal flux at the position of the pinhole into a thickness profile on the substrate, either planar or cylindrical, behind that pinhole. In the present paper, the thickness profile $\mathrm{T}(\mathrm{x})$ along a strip of approximately $0.5 \mathrm{~mm}$ is measured and converted into the angular flux $\Phi(\varphi)$ of impinging particles at the pinhole site using Eq.(3). The working principle (in this case for a cylindrical substrate) is sketched in figure 1a. It is a sealed (except the pinhole) cylindrically shaped chamber (figure 1b), and is connected to a stainless steel pipe with a KF40 linear feed-through. 

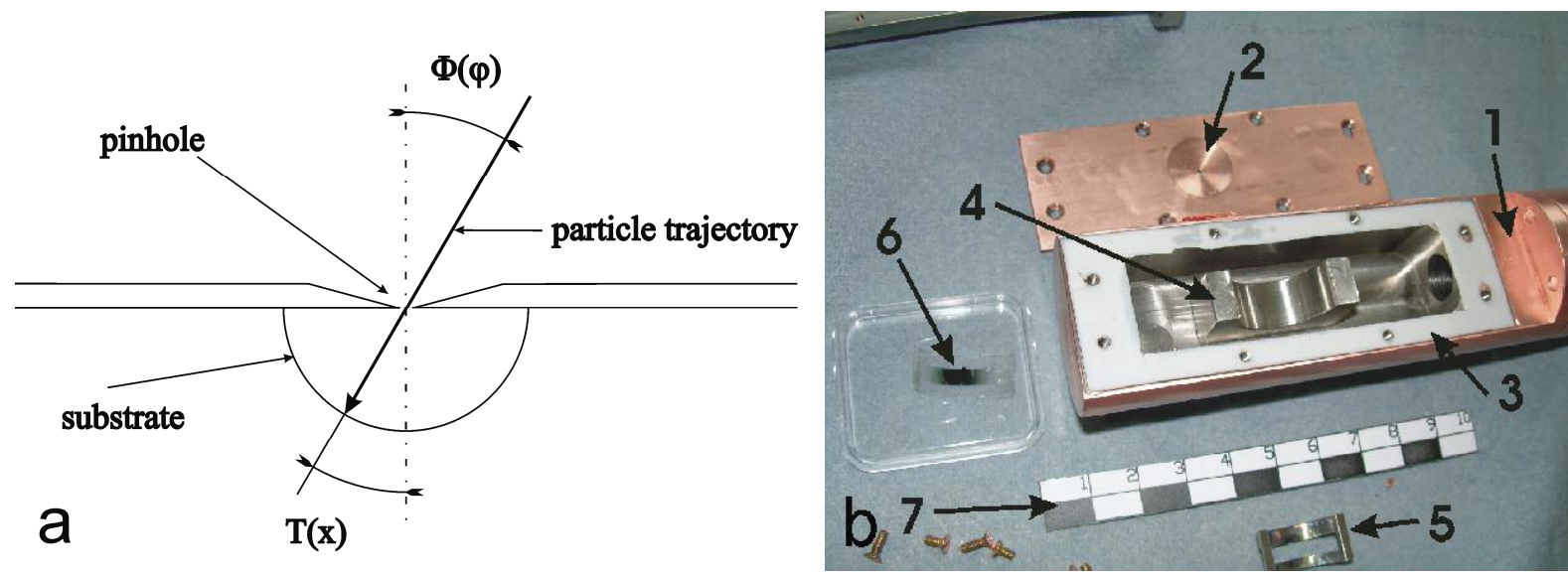

Figure 1. a) Sketch of the working principle of the pinhole camera with a cylindrical substrate holder. b) Picture of the MFM, indicated parts are: housing(1), pinhole (2), gasket(3), cylindrical substrate holder(4), cylindrical bracket(5), coated slide(6), measuring bar of $10 \mathrm{~cm}(7)$.

There is a MFM with the pinhole directed perpendicular to the feed-through axis (figure 1b), which can hold a cylindrical or a planar substrate holder, and one with the pinhole directed parallel to the feed-through axis, which can only hold a planar substrate holder.

The direct connection of the MFM to a pipe and a linear feed-through enhances its flexibility, as it can be mounted to every deposition chamber with a KF40 flange, and its position can be changed quickly. It also enables differential pumping of the MFM via the pipe (to increase the mean free path of particles inside the MFM), and the inlet of reactants directly into the MFM from outside the recipient. Some important data is given in Table 1

radius pinhole $\mathrm{r}$

$0.5 \mathrm{~mm}$

length pinhole $\mathrm{d}$

$0.2 \mathrm{~mm}$

Maximum angle error $\Delta \varphi$

$2.9^{\circ}$

Cover plate thickness

$2 \mathrm{~mm}$

Radius cylindrical substrate holder

$10 \mathrm{~mm}$

Distance pinhole to planar substrate

$10 \mathrm{~mm}$

Acceptance angle cylindrical

$75^{\circ}$

Acceptance angle planar

$45^{\circ}$

Tab.1 Technical Data MFM 


\section{Determination and simulation of the angular distribution of the metal flux}

To convert the thickness profile $\mathrm{T}(\mathrm{x})$ on the substrate into the angular flux $\Phi(\varphi)$ of impinging particles at the pinhole site, one has to take into account the geometry of the pinhole. The effective area of the pinhole decreases with increasing angle, an effect called vignetting $(\mathrm{V}(\varphi))$ (figure 2), thus also decreasing the number of passing particles.

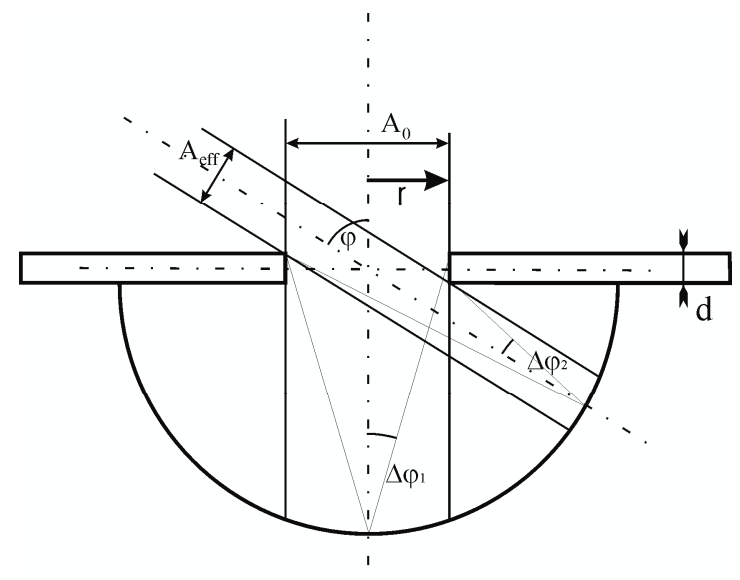

Figure 2. Vignetting: Two particles are entering the MFM at different angles $\varphi$; one perpendicular ( $\varphi$ $=0^{\circ}$; aperture $A_{0}$ of $r^{2} \pi$ ) and the second one at an angle $\varphi_{2}$ with a smaller aperture of $A_{\text {eff: }}$ The angle error $\Delta \varphi$ decreases at higher angles because of the decreasing aperture.

For a cylindrical pinhole (length d; radius $r$ ) $\mathrm{V}(\varphi)$ is described as the ratio of effective area $\mathrm{A}_{\text {eff }}$ seen by the tilted beam to the area of the pinhole $A_{0 .} V(\varphi)$ can be expressed as

$V(\varphi)=\frac{A_{e f f}}{A_{0}}=\frac{2 \cos (\varphi)}{\pi}\left[\frac{\pi}{2}-\arcsin \left(\frac{d \tan (|\varphi|)}{2 r}\right)-\left(\frac{d \tan (|\varphi|)}{2 r} \sqrt{1-\left(\frac{d \tan (|\varphi|)}{2 r}\right)^{2}}\right)\right]$

For a cylindrical shaped substrate, $\mathrm{T}(\mathrm{x})$ can be converted into $\Phi(\varphi)$ according to

$$
\Phi(\varphi) \propto \frac{T(x(\varphi))}{V(\varphi)}
$$

Aside from cylindrical substrates, also planar substrates were used. They were necessary, since the flexible substrates needed for the cylindrical substrate holder prohibit certain surface analysis techniques (e.g. thickness profile determination of oxides; see below). Compared to the cylindrical substrate, planar substrates do have the disadvantage that the distance between pinhole and substrate is angle dependant, and that the incidence angle on the substrate is not perpendicular anymore. 


\section{Determination and simulation of the angular distribution of the metal flux}

For planar substrates $\mathrm{T}(\mathrm{x})$ can be converted into $\Phi(\varphi)$ according to

$$
\Phi(\varphi) \propto \frac{T(x(\varphi))}{V(\varphi) \cdot \cos ^{3}(\varphi)}
$$

The resolution of the MFM is limited by two effects; one is the angular error $\Delta \varphi$ caused by the aperture (figure 2), the other is gas phase scattering inside the MFM, which can be lowered by differential pumping of the MFM.

Another limiting factor might be that only those particles which stick contribute to film growth and can therefore be measured. The sticking probability is determined by energy and incidence angle [17]. For the cylindrical shaped substrate the incidence angle is always perpendicular, therefore the sticking probability can be assumed to be 1 , even at higher energies. As mentioned above, the incidence angle on the planar substrate is not perpendicular. However, the sticking probability becomes angle dependant at higher angles (above $45^{\circ}$, which is the maximum acceptance angle of the MFM). Moreover, in the described experiments the particles which are not scattered, and therefore have higher energies, have typically incidence angles of $20^{\circ}$ and below. Therefore, even for the planar substrate the sticking coefficient can be assumed to be 1 .

Due to the pinhole geometry, the deposition rate on the substrate is drastically reduced (by a factor of approx. 0.01 and lower), thus increasing problems with residual gas incorporation. The reason is that the ratio of the impingement rates of the residual gas molecules $v_{g}[18]$ and of the sputtered particles $v_{d}$, given in (4), increases with decreasing deposition rate.

$$
\frac{v_{g}}{v_{d}}=\frac{m_{d} \cdot p}{a_{w} \rho \sqrt{2 \pi m_{g} k_{B} T}}
$$

$p=$ residual gas pressure $[\mathrm{Pa}]$

$m_{g}=$ molecular or atomic mass of the residual gas $[\mathrm{kg}]$

$m_{\mathrm{d}}=$ molecular or atomic mass of the sputtered material $[\mathrm{kg}]$

$k_{B}=$ Boltzmannconstant $\left(1.38 \cdot 10^{-23} \mathrm{JK}^{-1}\right)$

$T=$ temperature $[\mathrm{K}]$

$a_{w}=$ deposition rate $\left[\mathrm{ms}^{-1}\right]$

$\rho=$ density of deposited material $\left[\mathrm{kgm}^{-3}\right]$ 


\section{Determination and simulation of the angular distribution of the metal flux}

If one assumes a residual oxygen pressure of $10^{-5} \mathrm{~Pa}$ and a deposition rate of $5 \cdot 10^{-12} \mathrm{~ms}^{-1}$ of $\mathrm{Al}$ inside the MFM the ratio $\mathrm{v}_{\mathrm{g}} / \mathrm{v}_{\mathrm{d}}$ would be approximately 1 . Knowing that the oxygen incorporation coefficient during sputter deposition of reactive metals like $\mathrm{Al}$ and $\mathrm{Mg}$ can reach values up to $0.1-0.25[19,20]$, the formation of oxide layers seems unavoidable, even for a low residual gas pressure.

The thickness profiles on the substrates were determined via optical methods. For metallic layers the thickness was densitometrically determined via an optical scanner, as in the previous papers $[12,13]$. The transmissivity (figure 3) of the metallic layers was spatially resolved by a transmission scanner (reflecta CrystalScan 7200) and the transmissivity of the coated substrates was compared to that of an uncoated substrate. With the known transmissivity, the thickness of each point of the profile can be determined.

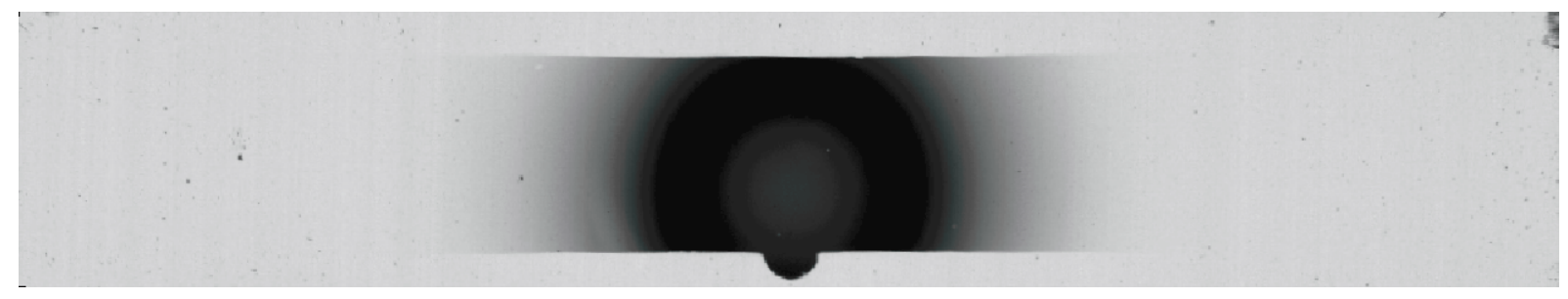

Figure 3. Grey scale image (green channel) of a scanned sample (Cu deposited on a flexible and transparent substrate).

The thickness profiles of the oxide layers were determined via a spatially resolved interferometric method especially designed for this task, where the oxide layer is deposited on a reflective substrate (e.g. polished silicon wafer) and its interference patterns are captured by a digital camera (figure 4a). The image is then colour split (figure $4 \mathrm{~b}-4 \mathrm{~d}$ ) and the profile determined for each colour individually (assuming a two beam interference). These three profiles are then averaged. For the interferometric method an Olympus Stylus 760 was used as digital camera. 


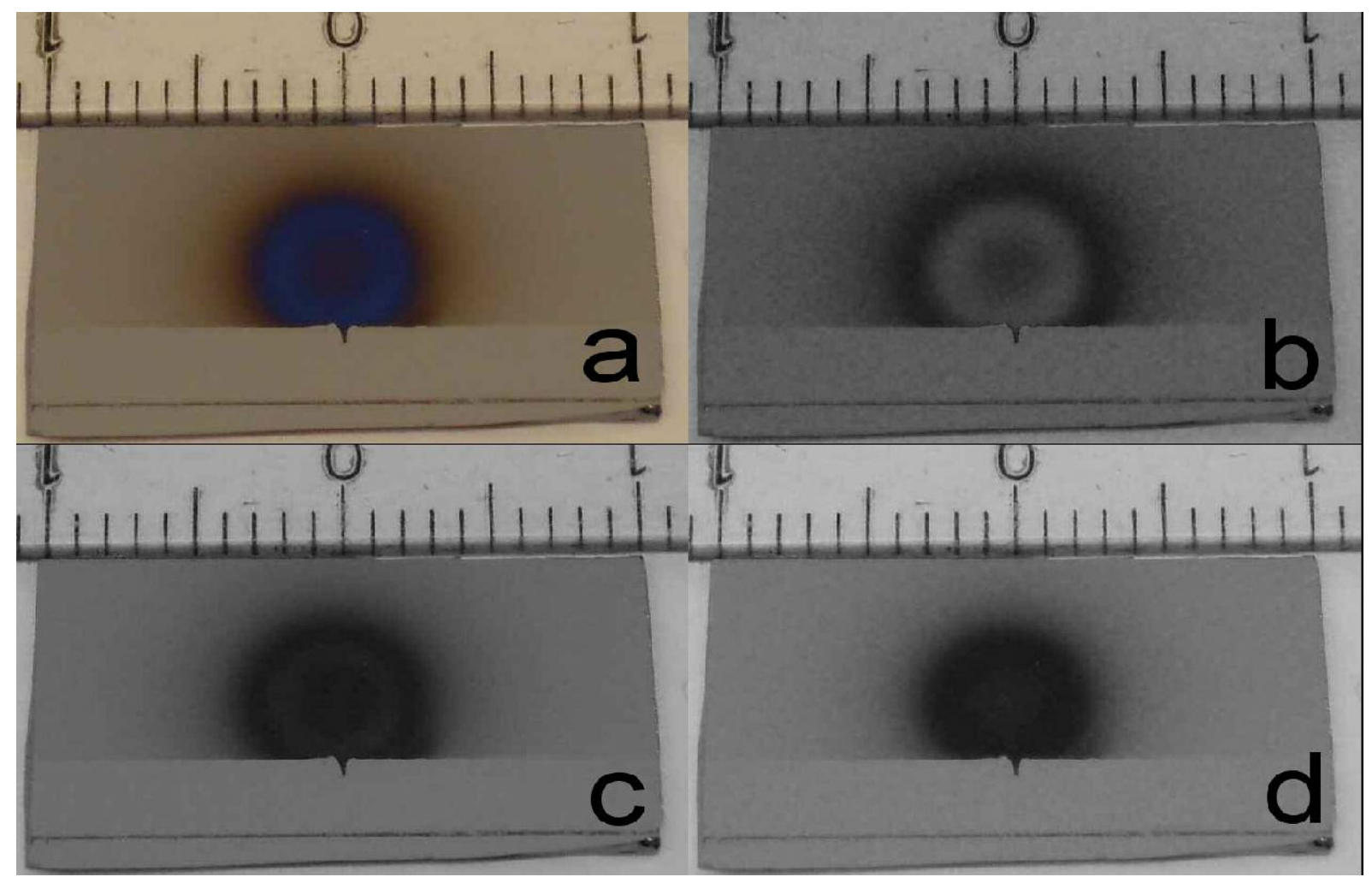

Figure 4 a) Image of a sample (Al deposited on polished silicon wafer) captured by a digital camera.

Greyscale images of the b) blue; c) green; d) red colour channel. The interference patterns are clearly visible.

\section{Experimental}

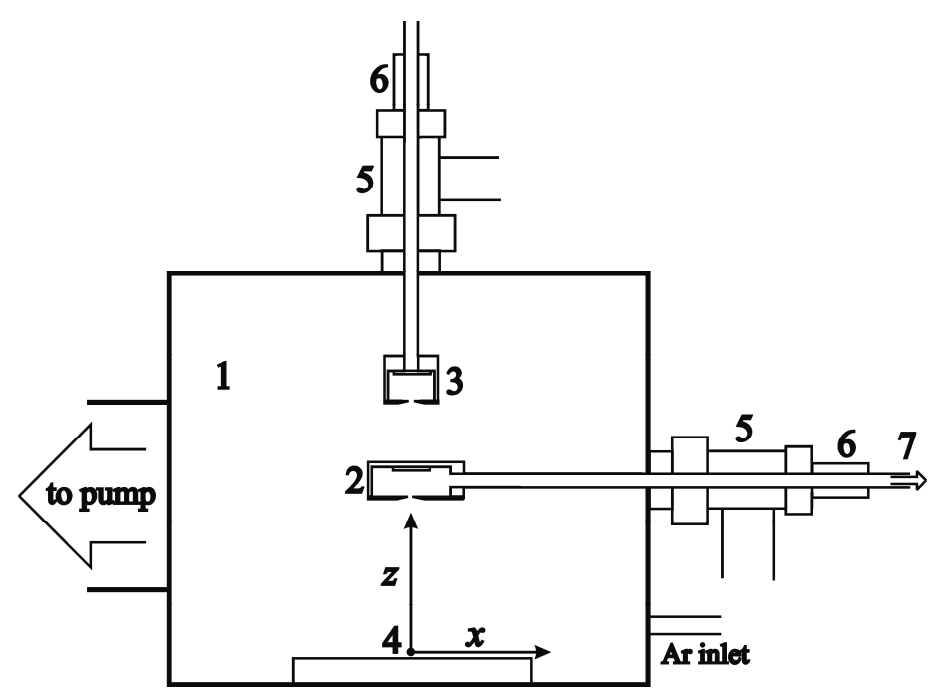

Figure 5. Experimental setup: vacuum chamber (1), MFM (with planar substrate) movable in x-axis (2), MFM (with planar substrate) movable in z-axis (3), target and origin of coordinates in its centre 


\section{Determination and simulation of the angular distribution of the metal flux}

(4), load-lock chamber (5), linear feed through for KF40 flange (6), pipe of the MFM leading outside the chamber, used for differential pumping and inlet of gas into the MFM (7).

The experimental set-up is depicted in figure 5. Experiments were performed in different vacuum chambers with base pressures of about $10^{-5} \mathrm{~Pa}$. A planar disc shaped magnetron source with a target diameter of $100 \mathrm{~mm}$ was used. The distance of the target centre to the area with the highest local sputter rate, called the racetrack, was $23 \mathrm{~mm}$. Angular measurements were carried out for $\mathrm{W}, \mathrm{Cu}, \mathrm{Ti}$, $\mathrm{Al}$ and $\mathrm{Mg}$ targets sputtered with Ar as working gas (inlet via a needle valve or a mass flow controller). Experiments were carried out for different pressures with the monitor mounted $95 \mathrm{~mm}$ centrally above the target, and also at a constant pressure of $0.5 \mathrm{~Pa}$ for different lateral (x-positions) and target substrate (z) distances. For the experiments with a different z-distance a small eccentricity of $5 \mathrm{~mm}$ was experimentally unavoidable, because the load lock was not right opposite the magnetron. A summary of the experimental parameters is given in Tab. 2 .

\begin{tabular}{ccc} 
Material & Pressures $[\mathrm{Pa}](\mathrm{x}=0 ; \mathrm{z}=95 \mathrm{~mm})$ & x-positions $[\mathrm{mm}](\mathrm{p}=0,5 \mathrm{~Pa}, \mathrm{z}=95 \mathrm{~mm})$ \\
\hline $\mathrm{Cu}$ & $0.3 ; 0.5 ; 0.7 ; 1 ; 3$ & $0 ; 11 ; 23 ; 35$ \\
$\mathrm{~W}$ & $0.3 ; 0.5 ; 0.7 ; 1 ; 3$ & $0 ; 11 ; 23 ; 35$ \\
$\mathrm{Al}$ & $0.3 ; 0.5 ; 0.7 ; 1$ & $0 ; 11 ; 23 ; 35$ \\
$\mathrm{Mg}$ & $0.1 ; 0.3 ; 0.5 ; 0.7 ; 1$ & $0 ; 11 ; 23 ; 35$ \\
$\mathrm{Ti}$ & $0.3 ; 0.5 ; 0.7 ; 1$ & z-distances $[\mathrm{mm}]($ eccentricity $\mathrm{x}=5 \mathrm{~mm} ; \mathrm{p}=0,5 \mathrm{~Pa})$ \\
\hline \multicolumn{2}{c}{$25 ; 50 ; 95 ; 125 ; 150$}
\end{tabular}

Tab.2 Parameters of the experiments.

The magnetron was operated at a constant DC discharge current of $0.3 \mathrm{~A}$ (experiments with variable pressure and lateral position) and $0.9 \mathrm{~A}$ (experiments with variable distance). $\mathrm{Cu}$ and $\mathrm{W}$ were deposited as metallic layers using the cylindrical substrate holder with transparent slides as substrates. $\mathrm{Al}$ and $\mathrm{Mg}$ were completely oxidized by the residual gas and were therefore deposited on silicon wafers in the planar substrate holder, to enable the profile determination via the interferometric method. Ti was only partially oxidized by the residual gas, hence a very small amount of oxygen was 


\section{Determination and simulation of the angular distribution of the metal flux}

inserted directly into the MFM (without influencing the sputtering process) to form an oxide layer, otherwise it was treated like $\mathrm{Al}$ and $\mathrm{Mg}$. Although the maximum acceptance angle of the cylindrical substrate is $75^{\circ}$, the experimental profiles were cut off at $45^{\circ}$, because the signal to noise ratio becomes large at higher angles.

\section{Simulations}

The metal flux towards the flux monitor was simulated using the MC-code SIMTRA [16], which tracks a representative set of individual particles in their movement through the vacuum chamber. By its working principle, first a sputtered particle is generated with initial position, energy and direction, sampled from given distribution functions. Here the ejection positions were taken from a measured erosion profile, neglecting the small influence of the target material on the racetrack shape. The initial energy distribution resulted from simulations using the binary collision code SRIM [21] with a constant incident ion energy corresponding to 75 percent of the discharge voltage $[22,23]$. Although SRIM also provides the initial angular distributions, these did not take the typical heart-like shape observed experimentally [24-28]. Hence, as was described in [16], initial angular distributions for $\mathrm{Cu}$, $\mathrm{Al}$ and $\mathrm{Ti}$ were instead reconstructed from deposition measurements, using the transport code in a reverse way. For $\mathrm{W}$ and $\mathrm{Mg}$ on the other hand, having no data available, the SRIM initial angular distributions were used. However, considering the geometry of the experimental setup, this parameter was expected and found to have a limited influence.

In the next step of the model the collisional transport of the sputtered particle through the gas phase is described. The background gas was assumed homogenous at temperature $\mathrm{T}=350 \mathrm{~K}$ and specified pressure $\mathrm{P}$. The assumption of an homogenous temperature distribution is valid, since the heating of the gas [29] and gas rarefaction [30, 31] are negligible at pressures below $1 \mathrm{~Pa}$. Collisions were modelled based on either quantum chemical ( $\mathrm{Cu}-\mathrm{Ar}, \mathrm{Al}-\mathrm{Ar}$ [32]) or screened Coulomb interaction potentials. Also the thermal motion of the background gas atoms was included. The sticking coefficient at the substrate was assumed to be unity.

The simulated configuration mimicked the experimental set-up, including the inside of the metal flux monitor. The angular distribution can be directly constructed from the particles arriving at the pinhole. 


\section{Determination and simulation of the angular distribution of the metal flux}

By continuing to track the particles through the MFM and following the experimental procedure, i.e. calculation of the deposition profile and applying vignetting correction, it is possible to gauge the influence of scattering in the flux monitor and the angular error due to the aperture.

\section{Results and discussion}

The influence of the deposition pressure on the angular distribution $\Phi(\varphi)$ at a substrate positioned at z $=95 \mathrm{~mm}$ and $\mathrm{x}=0 \mathrm{~mm}$ (central above the target) is shown in figures $6 \mathrm{a}-\mathrm{d}$ for $\mathrm{W}, \mathrm{Cu}, \mathrm{Ti}$ and $\mathrm{Al}$ respectively. The calculated angular distribution with neglected gas phase scattering $(\mathrm{p}=0 \mathrm{~Pa})$ is plotted for W. Clearly visible in figure 6 is the broadening of the angular distribution with increasing pressure, reproduced well by the SIMTRA simulations. With some exceptions this is also the trend observed in the material dependence. The lighter elements are averagely scattered over larger angles, leading to an increase of the fraction of the flux arriving at higher incident angles. A good parameter to quantify the amount of gas scattering is the ratio $\mathrm{R}_{\min } / \mathrm{R}_{\max }$ of the local minimum in the centre of the target and the maximum at the racetracks (indicated in figure 6 a for $0.3 \mathrm{~Pa}$ ). Since $\Phi(\varphi)$ is symmetrical in this case, because the MFM is centrally above the target, the results are plotted from $\varphi=0^{\circ}$ to $\varphi=$ $45^{\circ}$.

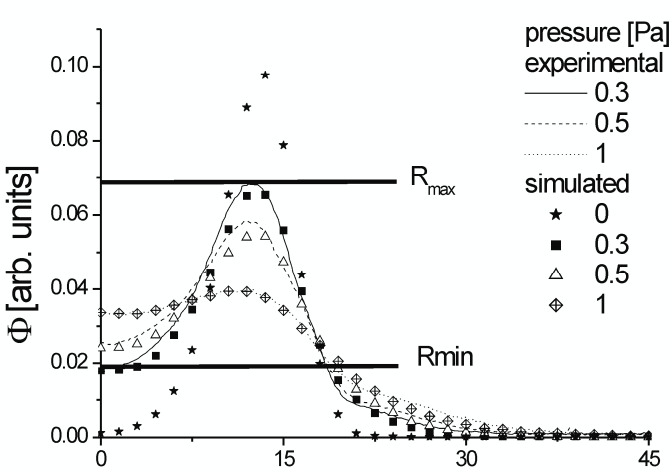

a

$\varphi\left[{ }^{\circ}\right]$

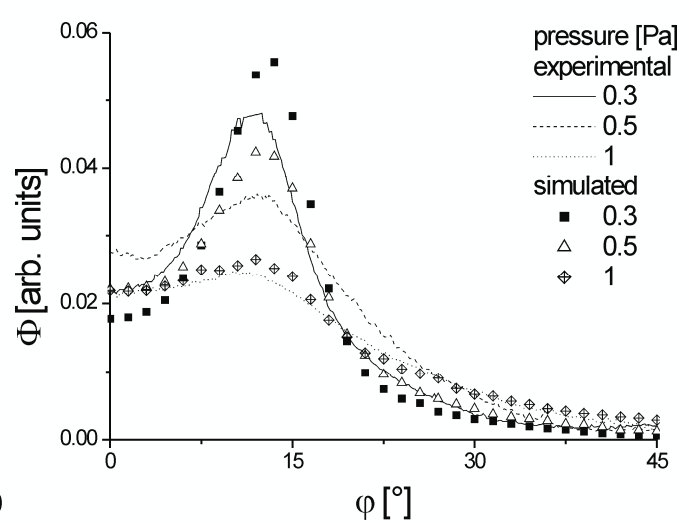

b 

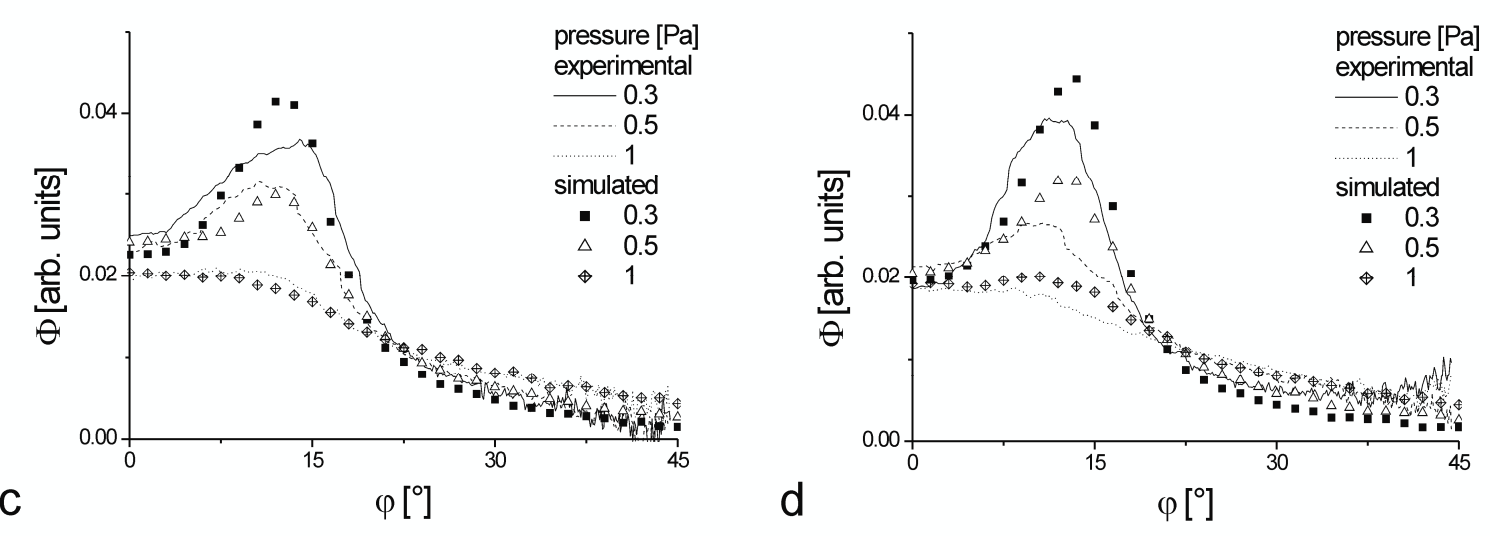

Figure 6: Angular distribution $\Phi(\varphi)$ of the metal flux at a substrate $95 \mathrm{~mm}$ above the target surface as function of the discharge pressure for a) $\mathrm{W}, b) \mathrm{Cu}, c) \mathrm{Ti}, d) \mathrm{Al}$. For $\mathrm{W}$ the angular distribution at $p=$ O Pa (no gas phase scattering) is also shown. The angular distributions have local minimum at the centre $\left(\varphi=0\right.$, value $\left.R_{\min }\right)$, and a maximum at the racetracks (value: $\left.R_{\max }\right)$.

The influence of gas phase scattering inside the MFM was investigated with simulations. Scattering inside the monitor broadens the angular profile somewhat, although the effect is limited at all pressures. At low pressure the arriving flux is directional, but the scattering probability remains low so the profiles stay relatively intact. At higher pressure simulations showed significant scattering in the monitor, but due to scattering in the vacuum chamber, the arriving flux at the pinhole is already randomized to such a degree that the extra scattering in the monitor only has a limited influence on the resulting deposition profile. This indicates that, even without differential pumping, the flux monitor allows accurate measurements of the arriving angular distribution.

Figure 7 shows the influence of the $\mathrm{x}$ (lateral) position on the angular distribution of the arriving metal flux for $\mathrm{Cu}$ as example. The angular distribution shifts and becomes asymmetric. Although the effect is mainly geometrical, an accurate quantification can help in improving for instance the uniformity of films deposited under conditions were the arriving angular distribution influences the microstructure. 


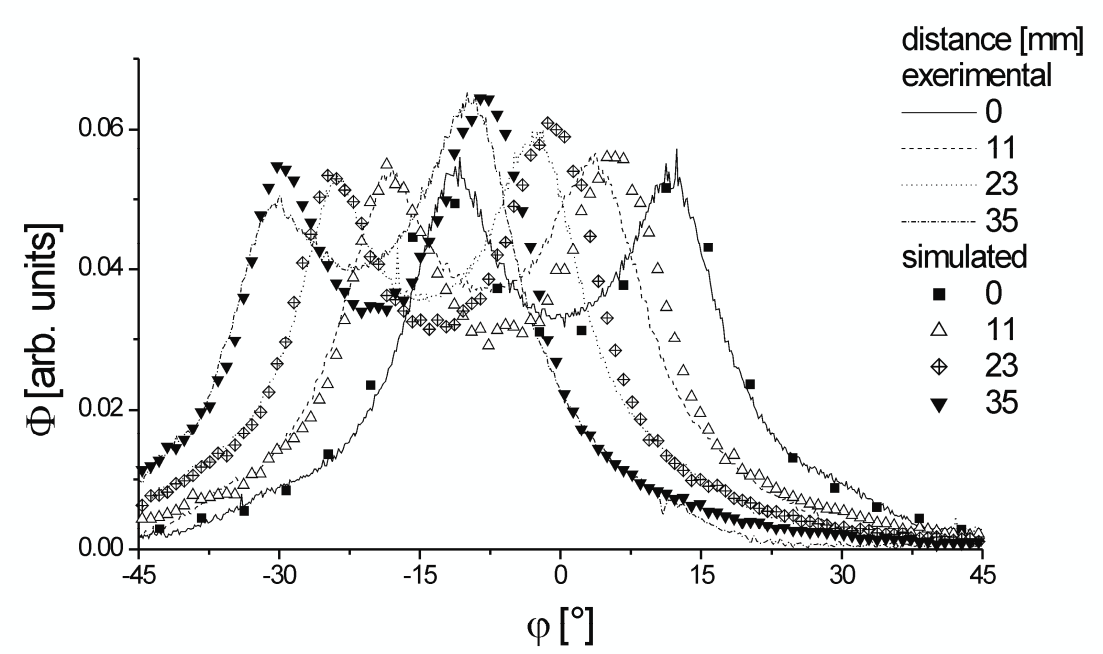

Figure 7: Angular distribution $\Phi(\varphi)$ of the metal flux at a substrate $95 \mathrm{~mm}$ above the target surface as function of the $x$ (lateral)-position for Cu sputtered at a pressure of $0.5 \mathrm{~Pa}$.

The results of the series with different $z$-distances (at a constant lateral eccentricity $x=5 \mathrm{~mm}$ ) are shown in figure 8 with the MC-simulation for comparison. As expected $R_{\min } / R_{\max }$ increases with the distance. Since the distributions are not symmetrical, due to the eccentricity, the results are plotted from $-45^{\circ}$ to $+45^{\circ}$.

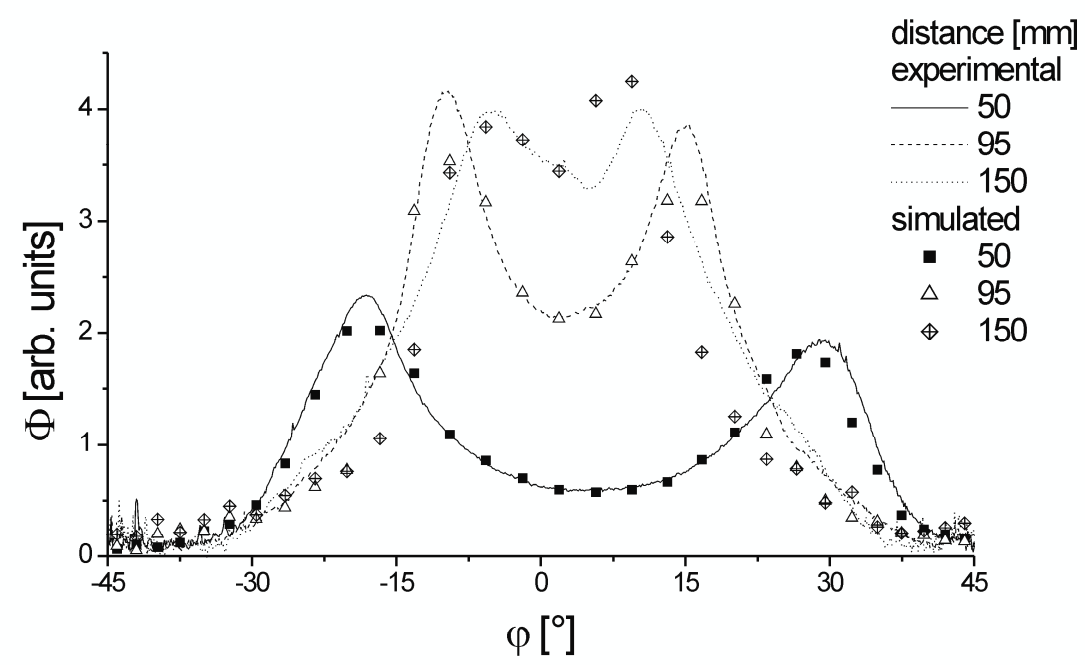

Figure 8 Angular distribution of copper as function of the distance $z$ of the substrate from the target.

Condition were $p=0.5 P a, x=0.5 \mathrm{~mm}$ (eccentricity). 


\section{Determination and simulation of the angular distribution of the metal flux}

Due to collisions with the working gas, the sputtered particles will loose energy and direction. Hence, due to collisions, the sputtered particles will get more and more thermalized. This degree of thermalization (the point where the sputtered particles can be treated as a Maxwellian gas, i.e. thermal energy and random direction) was given by Gras-Marti [33] as $P_{\text {therm }}$ (5). The analytical model of the calculation assumed a continuous slowing down of particles along straight line trajectories with a velocity proportional energy loss per unit path length.

$$
P_{\text {therm }}=\frac{x^{2}}{R(U)^{2}+x^{2}}
$$

where $\mathrm{x}$ is the distance target-MFM, $R(U)(6)$ is the path length of a particle with initial energy $U$ (surface binding energy) until thermalization is reached.

$$
R(U)=A \cdot U^{1 / 2}
$$

The energy independent coefficient $A$ is given by

$$
A \cong \frac{0.012}{M_{g}{ }^{1 / 2}}\left(1+\frac{1}{\mu}\right)^{1 / 2}\left(1+\mu^{2 / 3}\right)^{3 / 4} \frac{T(K)}{p(P a)}
$$

with:

$\mu=M_{g} / M_{s}$

$M_{g}=$ mass of gas [amu]

$M_{s}=$ mass of target material [amu]

$T=$ temperature $[300 \mathrm{~K}]$

$\mathrm{U}=$ surface binding energy $[\mathrm{eV}]$

This probability of thermalization $P_{\text {therm }}$ can be compared to the ratio $\mathrm{R}_{\min } / \mathrm{R}_{\max }$ of the local minimum in the centre of the target and the maximum at the racetracks (indicated in figure 6a). Indeed, the flux in the center should be zero when there is no gas scattering $\left(R_{\min } / R_{\max }=0\right)$ and should be maximal when there is a lot of gas phase scattering $\left(R_{\min } / R_{\max }=1\right)$. This relation between $P_{\text {therm }}$ and $R_{\min } / R_{\max }$ is shown in figure 9. It can be concluded that the MFM allows measuring the degree of thermalization of the sputtered particles. 


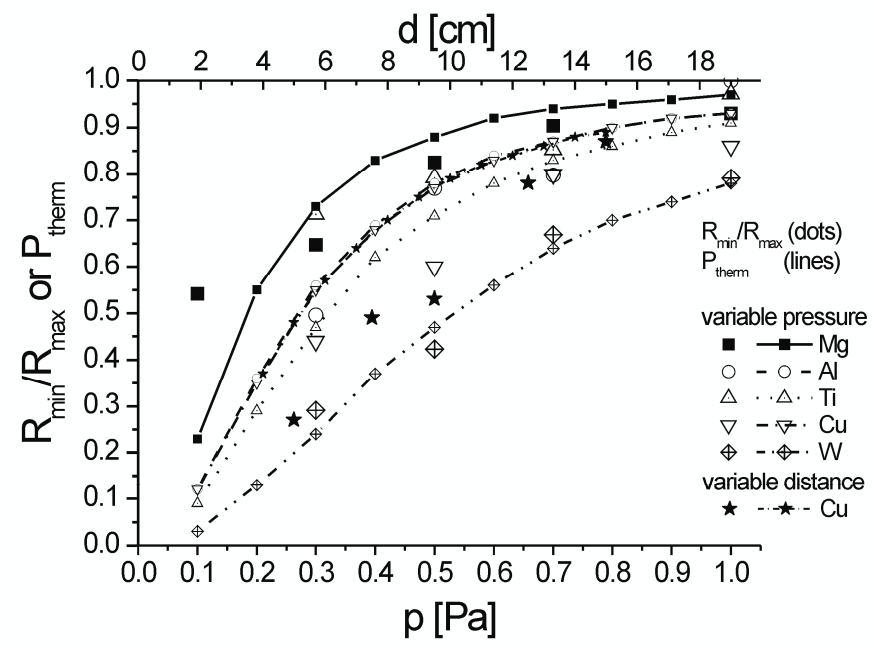

Figure 9. Comparison between $R_{\min } / R_{\max }$ (observed) and $P_{\text {therm }}$ (calculated) for all investigated target materials at different deposition pressures and for $\mathrm{Cu}$ with variable distances.

\section{Conclusions}

The MFM allows measuring the angular distribution of the metal flux. Also information on the number of thermalized neutrals can be obtained from the measured angular distribution. The different presented methods for thickness determination with a good lateral resolution allow the MFM to be used for a wide variety of target materials. It was demonstrated that $\Phi(\varphi)$ is not isotropic for the usual deposition parameters. The experimentally obtained results were compared to simulations and a good match was found. It was also shown that the ratio $R_{\min } / R_{\max }$ is correlated to the degree of thermalization of the metal flux. Therefore the degree of thermalization can be estimated for given process parameters with one measurement.

\section{Acknowledgments}

This work was supported by the Institute for the promotion of Innovation by Science and Technology in Flanders (IWT) under Grant Nr. SBO-060030.

\section{References}


Determination and simulation of the angular distribution of the metal flux

[1] Bales G S, Bruinsma R, Eklund E A, Karunasiri R P U, Rudnick J, Zangwill A 1990 Science 249 $264-268$

[2] Bales G S, Zangwill A 1991 J. Vac. Sci. Technol. A 9 (1) 145-149

[3] Pelliccione M, Karabacak T, Gaire C, Wang G C, Lu T M 2006 Phys. Rev. B 74125420

[4] Blech I A, Vander Plas H A 1983 J. Appl. Phys. 54 (6) 3489-96

[5] Robbie K, Brett M J 1997 J. Vac. Sci. Technol. A 15 (3) 1460-65

[6] Lugscheider E, von Hayn G 1999 Surf. Coat. Technol. 116-119 568-572

[7] Wang L, Clancy P 2001 Surf. Sci. 473 25-38

[8] Mahieu S, Ghekiere P, Depla D, De Gryse R 2006 Thin Solid Films 515 1229-49

[9] Mahieu S, Ghekiere P, Dewinter G, De Gryse R, Depla D, Lebedev O I 2005 Solid State Phenomena 105 447-452

[10] Thornton J A, Hoffman D W 1981 J. Vac. Sci. Technol. 18(2) 203-207

[11] Swan S 1987 J. Vac. Sci. Technol. A 5 (4) 1750-54

[12] Eisenmenger C, Bergauer A, Bangert H, Bauer W 1994 J. Vac. Sci. Technol. A 12(2) 536-541

[13] Eisenmenger C, Beyerknecht R, Bergauer A, Bauer W, Betz G 1995 J. Vac. Sci. Technol. A 13(5) $2435-43$

[14] Feddes B, Wolke J G C, Jansen J A, Vredenberg A M 2003 J. Appl. Phys. 93 (1) 662-670

[15] Panjan M, Cremer R, Fuss H G, Panjan P, Cekada M The use of camera obscura in sputter deposition, Vacuum (2009), doi:10.1016/j.vacuum.2009.04.069. In Press

[16] Van Aeken K, Mahieu S and Depla D 2008 J. Phys. D: Appl. Phys. 41205307

[17] Zhou X W, Wadley H N G 1999 Surf. Sci. 431 58-73

[18] Maissel L, Glang R 1970 Handbook of Thin Film Technology (New York, McGraw-Hill) pp 1-21; ISBN 978-0070397422

[19] Leroy W P, Mahieu S, Persoons R, Depla D, accepted for publication in Plasma Processes and Polymers 10.1002/ppap.200932401

[20] Leroy W P, Mahieu S, Persoons R, Depla D, accepted for publication in Thin Solid Films 10.1016/j.tsf.2009.07.190

[21] The Stopping and Range of Ions in Matter 2006, available at http://www.srim.org/ 
Determination and simulation of the angular distribution of the metal flux

[22] Czekaj D, Goranchev B, Hollmann E K, Volpyas V A, Zaytsev A G 1991 Vacuum 42, 43

[23] Goeckner M J, Goree J A, and SheridanT E 1991 IEEE T. Plasma Sci. 19, 301

[24] Ramos R, Cunge G, Touzeau M, Sadeghi N 2008 J. Phys. D: Appl. Phys. 41, 152003

[25] Yamamura Y and Ishida M 1995 J. Vac. Sci. Technol. A 13101

[26] Goehlich A, Gillman D and Döbele H F 2001 Nucl. Instrum. Methods Phys. Res. B 179351

[27] Yamamura Y 1982 Nucl. Instrum. Methods 194515

[28] Tondu T 2005 PhD Thesis SUPAERO Engineering School, Toulouse, France

[29] Depla D Mahieu S 2008 Reactive Sputter Deposition (Berlin Heidelberg, Springer-Verlag) pp 120; ISBN 978-3540766629

[30] Palmero A, Rudolph H, Habraken F H P M 2006 Appl. Phys. Lett. 89211501

[31] Palmero A, Rudolph H, Habraken F H P M 2007 J. Appl. Phys. 101083307

[32] Kuwata K T, Erickson R I, Doyle J R 2003 Nucl. Instrum. Methods Phys. Res. B 201 566-570

[33] Gras-Marti A, Valles-Abarca J A 1983 J. Appl. Phys. 54(2) 1071-75 